\section{A Salmonella enterica subsp. enterica serovar Enteritidis foodborne outbreak after consumption of homemade lasagne}

Walter Vencia, ${ }^{1}$ Grazia Rosaria Gariano, ${ }^{1}$ Daniela Manila Bianchi, ${ }^{1}$ Fabio Zuccon, ${ }^{1}$ Marco Sommariva, ${ }^{2}$ Bovannrith Nguon, ${ }^{3}$ Aurelio Malabaila, ${ }^{4}$ Silvia Gallina, ${ }^{5}$ Lucia Decastelli ${ }^{1}$

1'Division of Food Control and Hygiene of Productions, Institute for Experimental Veterinary Medicine of Piedmont, Liguria and Aosta Valley, Turin; ${ }^{2}$ Institute for Experimental Veterinary Medicine of Piedmont, Liguria and Aosta Valley, Novara; ${ }^{3}$ Department of Prevention, Local Health Unit of Biella, Biella; ${ }^{4}$ Laboratory of Analysis, Degli Infermi Hospital, Biella; ${ }^{5}$ Reference Centre for Salmonella Typing - CeRTiS, Institute for Experimental Veterinary Medicine of Piedmont, Liguria and Aosta Valley, Turin, Italy

\section{Abstract}

In the latest year, and also in 2013, Salmonella was the most frequently detected causative agent in foodborne outbreaks (FBOs) reported in Europe. As indicated in EFSA report (2015) the serotypes mostly associated to FBOs are $S$. Typhimurium and Enteritidis; while Salmonella Typhimurium is generally associated with the consumption of contaminated pork and beef, FBOs due to Salmonella Enteritidis are linked to eggs and poultry meat. In this study it is described the investigation of a domestic FBO involving four adults and linked to homemade lasagne. Investigations were performed to determine the relatedness of Salmonella strains, identify the sources of infection, and trace the routes of Salmonella contamination in this FBO. Salmonella strains were isolated in 3 out of 4 patient stool samples and from lasagne and all of them were serotyped as $S$. Enteritidis. Pulsed-field gel electrophoresis (PFGE) analysis revealed the genotypical similarity of all the strains. Although serotyping and PFGE analysis identified the common food source of infection in this FB0, it was not possible to determine how or at what point during food preparation the lasagne became contaminated with Salmonella.

\section{Introduction}

Salmonella was the most common cause of foodborne outbreaks (FBOs) in the European Union (EU) in 2013, with 1168 FBOs of human salmonellosis reported by 22 member states, accounting for $22.5 \%$ of all notified outbreaks of foodborne illness in the EU according to the European Food Safety Authority (EFSA, 2015)

The genus Salmonella, which is closely related to the genus Escherichia, groups Gram-negative, non-spore-forming, is rodshaped bacteria belonging to the Enterobacteriaceae family. Two species are distinguished: $S$. enterica, classified into six subspecies (Grimont et al., 2007), and S. bongori. Salmonella enterica subspecies enterica is an intracellular pathogen of warm-blooded mammals comprising over 2500 serovars (Baker and Dougan, 2007). Some Salmonella serovars, such as $S$. enterica serovar Enteritidis and $S$. enterica serovar Typhimurium, are non-specific host pathogens that can colonise a broad range of animals, rarely causing clinical manifestations. In humans, however, they can lead to gastroenteritis or occasionally septicemia (Galanis et al., 2006). The serovars most frequently associated with human illness in the EU are $S$. Enteritidis (39.5\%), S. Typhimurium (20.2\%), and monophasic $S$. Typhimurium (8.6\%) (EFSA, 2015).

Salmonella is the most common causative agent, reason for hospitalisation, and cause of death tracked by the Foodborne Diseases Active Surveillance Network (FoodNet) (CDC, 2011). Beyond their health effects, foodborne illnesses may cause emotional and economic hardship. Salmonella alone is responsible for approximately 1 million foodborne infections (Scallan et al., 2011) and it costs 365 million \$ in direct medical expenditures annually (US Department of Agriculture, 2011).

Common salmonellosis symptoms include diarrhea, fever, and abdominal cramps beginning 12 to 72 hours after the consumption of contaminated food or beverages (Shariat et al., 2013). More often, human $S$. Typhimurium cases are associated with the consumption of contaminated pork and beef, whereas $S$. Enteritidis cases are linked to eggs and poultry meat. Subclinical infection in animals leads to herd or flock contamination, with intermittent or persistent shedding of bacteria (EFSA, 2013).

In laboratory testing for Salmonella enteri$c a$, isolates are identified and typed by several phenotypic methods, including biochemical profiling, serotyping, and phage typing. Standard serotyping methods, based on the detection of somatic (0) and flagellar (H) antigens, are tedious and time-consuming.
Correspondence: Walter Vencia, Division of Food Control and Hygiene of Productions, Institute for Experimental Veterinary Medicine of Piedmont, Liguria and Aosta Valley, via Bologna 148, 10154 Turin, Italy.

Tel. +39.011.2686.233 - Fax: +39.011 .2473450 .

E-mail:walter.vencia@izsto.it

Key words: Salmonella spp.; Foodborne outbreak; Pulsed-field gel electrophoresis.

Contributions: the authors contributed equally.

Conflict of interest: the authors declare no potential conflict of interest.

Received for publication: 26 February 2015. Revision received: 16 April 2015.

Accepted for publication: 17 April 2015.

This work is licensed under a Creative Commons Attribution-NonCommercial 3.0 International License (CC BY-NC 3.0).

(C) Copyright $W$. Vencia et al., 2015

Licensee PAGEPress, Italy

Italian Journal of Food Safety 2015; 4:5127

doi:10.4081/ijfs.2015.5127

Although they lack the capacity to fingerprint strains in a sensitive manner, they remain useful in surveillance programmes (Herikstad et al., 2002).

More recently, different genotyping methods have been developed for the genetic discrimination of Salmonella isolates in outbreaks, such as multilocus sequence typing, multilocus variable-number tandem-repeat analysis, next generation sequencing. Pulsedfield gel electrophoresis (PFGE) was adopted for Salmonella surveillance and outbreak research in the 1990s. Because of its remarkable discriminatory power and high reproducibility, it has been successfully used in typing Salmonella strains isolated from human patients, foods and feed (Zou et al., 2013). Although PFGE is also labour-intensive, public health surveillance laboratories use it to determine strain relatedness and to confirm FB0s. Indeed, the US CDC recommends PFGE as the gold standard method for molecular characterisation in outbreak investigations.

In this paper it was reported the investigation of a household/domestic kitchen FBO of salmonellosis occurred in Biella on the $15^{\text {th }}$ of January 2010 linked to homemade lasagne. Microbiological, serological, and molecular assays on Salmonella isolates from human and food samples were performed to determine the relatedness of the implicated Salmonella strains, identify the sources of infection, and trace the routes of Salmonella contamination in this circumscribed FBO. The study was conducted in collaboration between the Food Control Laboratory at the 
Institute for Experimental Veterinary Medicine of Piedmont, Liguria and Aosta Valley, Turin, the Biella Department of Public Health Prevention, and the Hospital of Biella Ospedale degli Infermi.

\section{Materials and Methods}

\section{Epidemiological investigation}

Four adults ( 1 man and 3 women; age range, 45-61 years) sought medical attention at the Emergency Room of the Degli Infermi Hospital, Biella because of gastrointestinal symptoms on the $16^{\text {th }}$ of January 2010 . From initial accounts, the physicians on duty at the time suspected a domestic FBO. All four persons were hospitalised, and the local Public Health and Prevention Department was notified. An epidemiological investigation was initiated. A standardised questionnaire was administered by the public health officials in order to obtain demographic and clinical data, history of recent travel, food and water consumption, exposure to animals, and other illness during the week before becoming ill. In the interviews, the patients stated that they had consumed a homemade baked lasagne dish consisting of boiled pasta in which cheese and tomato sauce are mixed and then cooked in the oven. Then, a mixture of raw eggs was added to homemade lasagne and left in switched off oven until the evening. Before eating, the homemade lasagne was reheated for a short time. In addition, the patients had also eaten salad, turkey meat, and cake.

People who consumed common meal on the $15^{\text {th }}$ of January 2010 in Biella, who had gastro enteric symptoms from $15^{\text {th }}$ to $16^{\text {th }}$ of January, had not gastro enteric symptoms before the $15^{\text {th }}$ of January 2010.

\section{Microbiological investigation}

Stool samples were collected from all patients. Only residues of the baked lasagne could be collected for sampling and subse- quent analysis. The food sample was analysed to identify Salmonella spp.; the stool samples were tested for Salmonella, Shighella and Campylobacter.

Salmonella from the food sample was isolated according to ISO 6579:2002/COR 1:2004 (ISO, 2004). This method entails several steps: pre-enrichment in non-selective liquid medium (buffered peptone water; Biokar Diagnostic, Beauvais Cedex, France); enrichment in two selective liquid media [Rappaport-Vassiliadis medium with soya broth (Microbiol Diagnostici, Cagliari, Italy) and Muller-Kauffmann tetrathionate novobiocin - MKTTn - broth (LIOFILCHEM srl, Roseto, TE, Italy)]; and plating on xylose lysine deoxycholate agar (XLD) (Microbiol Diagnostici) and brilliant green agar (BGA) (Microbiol Diagnostici).

An internal method for detecting Salmonella, Shighella and Campylobacter was carried out on the stool samples. Specifically, for the isolation of Salmonella and Shighella have been used Hektoen Enteric Agar (Oxoid, Rodano, MI, Italy), Mac Conkey Agar (Oxoid) and Salmonella Shighella Agar (Oxoid); for the isolation of Campylobacter the stool samples were analyzed using Campylobacter Species Ag Rapid Test (Li StarFish S.r.l., Cernusco, MI, Italy) and Campylobacter selective agar (BioMérieux, Marcy l'Etoile, France).

All isolated strains were identified by conventional biochemical methods (API 20E; BioMériux) and the Salmonella spp. were serotyped according to the Kauffman-WhiteLe Minor classification scheme (Kauffmann, 1966; Le Minor and Popoff, 1987, Grimont and Weill, 2007).

The isolates were processed according to the PFGE protocol described by the US Centers for Disease Control and Prevention (CDC, 2013) using XbaI (Carlo Erba Reagents Srl, Cornaredo, MI, Italy) and BlnI (Roche Diagnostics Corporation, Indianapolis, IN, USA) restriction enzymes. PFGE using a sin- gle restriction enzyme $(X b a \mathrm{I})$ is a standard method for genotyping $S$. Enteritidis (Dewaele et al., 2012). Thus, the ability to deduce the serotype of a Salmonella isolate based on its PFGE profile provides an alternative method for screening and identifying Salmonella serotypes (Zou et al., 2010).

The PFGE fingerprint patterns were analysed with BioNumerics software (version 7.1; Applied Maths, Sint-Martins-Latem, Belgium) and the PFGE patterns were normalised by interpolation to the nearest reference lane (Salmonella Braenderup strain ATCC H9812). For comparing the PFGE profiles, values of optimization of $1.0 \%$ and position tolerance of $1.0 \%$ were applied to both enzymes. Dice similarity coefficients were calculated on the basis of pairwise comparisons of the PFGE profiles. The complete linkage algorithm was used for dendrogram construction. Isolates were considered identical if their profiles showed 100\% similarity.

\section{Results}

Epidemiological investigation revealed the main symptoms of nausea (50\%), abdominal cramps (75\%), diarrhea (100\%), vomiting (100\%), fever (100\%), and low back pain (25\%). The symptoms began 5 hours after eating the meal in the first case and 9 hours later in the last case. During the interview no history of recent travel, food and water consumption, exposure to animals, and other illness during the week before becoming ill was reported. A participant cooked the baked lasagne at the dinner and all the people ate the lasagne. The eggs were bought at the market.

Salmonella spp. was isolated in three patients (stool samples) and in the baked lasagne (residues stored in the refrigerator); one patient was tested negative for all pathogens. $S$. Enteritidis was identified by serotyping in the four isolated strains. Four $S$.

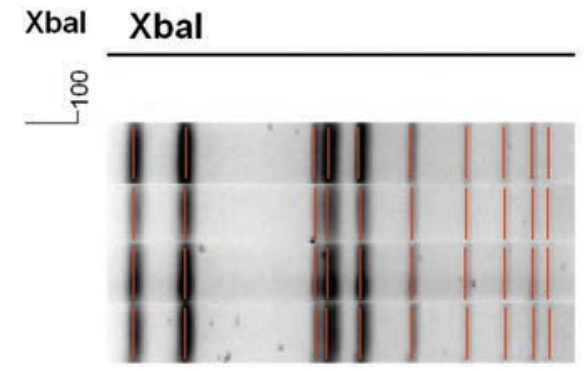

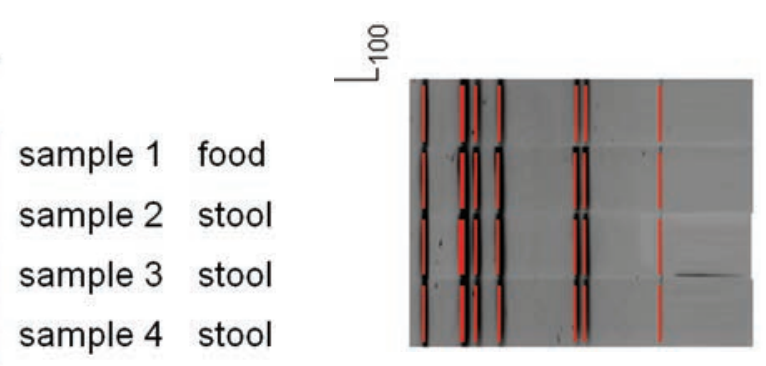

sample 3 Blnl sample $2 \mathrm{BInl}$ stool sample $1 \mathrm{BInl}$ food sample 4 BInl stool
Figure 1. Single enzyme restriction analyses by $X b a I$ distributed the four isolates into one cluster.
Figure 2. DNA digestion with restriction enzyme $B \ln I$ generated one cluster. 
Enteritidis isolates were genotyped by PFGE using $X b a \mathrm{I}$ and $B \ln \mathrm{I}$ restriction enzymes, which yielded consistent reproducible fingerprints. Single enzyme restriction analyses by $X b a$ I distributed the four isolates into one cluster (Figure 1). DNA digestion with restriction enzyme BlnI also generated one cluster (Figure 2). Overall, the $S$. Enteritidis population appeared to be genotypically similar.

\section{Discussion}

$S$. Enteritidis is the major serovar associated with human salmonellosis linked to the consumption of contaminated poultry products, including eggs (Braden, 2006; Much et al., 2009). In 2013207 strong evidence outbreaks were attributed to the consumption of eggs and egg products and 59.9\% of FBOs were caused by $S$. Enteritidis (EFSA, 2015). A 2002-2003 US case-control study reported that a significant risk factor of Salmonella infections (OR 2.7, 95\% CI 1.1-3.9) is consuming undercooked eggs or egg-containing dishes inside the home (Middleton et al., 2014). The household/domestic kitchen remains the most commonly reported setting in European FBOs (38.5\%) followed by the public/commercial kitchens (restaurant, café, pub, bar, hotel categories) in 2013 (22.2\%) (EFSA, 2015).

The domestic FB0 outbreak described here was linked to the homemade lasagne contaminated with Salmonella spp. We identified a common source of infection by using PFGE, one of the most important molecular characterization methods of diagnostic testing to compare bacteria strains. How and at which point during food preparation the lasagne became infected with Salmonella cannot be definitively determined, but the data suggest opportunities for person to food, food to food, and equipment to food cross-contamination.

Generating a hypothesis about the likely sources, the most probable is that the eggs used in preparing the lasagne were probably contaminated with Salmonella Enteritidis and their adding after cooking process is responsible of the contamination of RTE product. Indeed the reheating process before consumption normally applied at low temperature is not enough to kill the bacteria and might even to promote the bacterial growth.

A plausible alternative hypothesis is that the lasagne was contaminated with Salmonella Enteritidis after cooking due to cross-contamination. Indeed, one of the most common causes of FBOs is cross-contamination during food handling due to the use of improperly washed utensils and handling by ill or healthy carriers.

\section{Conclusions}

Serotyping according to the KauffmanWhite-Le Minor classification scheme and PFGE analysis allowed identifying the source of food poisoning in this domestic FBO. However, it was arduous to single out which ingredient of the meal was the source of Salmonella contamination. Collaborative efforts by the hospital physicians, public health department officials, and laboratories using different laboratory analysis methods during the outbreak investigation were essential for identifying the causative agent. Good sanitation, safe food handling and preparation practices in the entire food chain are key to preventing foodborne illness. Consumers should be made aware of the risks of FBO and learn how to minimise their chances of becoming ill by considering food safety at each step, from food purchase, to cooking, cleaning, and storing leftovers appropriately (International Food Information Council Foundation, 2014).

\section{References}

Baker S, Dougan G, 2007. The genome of Salmonella enterica serovar Typhi. Clin Infect Dis 45(Suppl.1):S29-33.

Braden CR, 2006. Salmonella enterica serotype Enteritidis and eggs: a national epidemic in the United States. Clin Infect Dis 43:512-7.

CDC, 2011. Vital signs: incidence and trends of infection with pathogens transmitted commonly through food. Foodborne diseases active surveillance network, 10 U.S. Sites, 1996-2010. Centers for Disease Control and Prevention, Atlanta, GA, USA. Available from: http://www.cdc.gov/mmwr/preview/mmwr $\mathrm{html} / \mathrm{mm} 6022 \mathrm{a} 5 . \mathrm{htm}$

CDC, 2013. Standard operating procedure for PulseNet PFGE of Escherichia coli 0157:H7, Escherichia coli non-0157 (STEC), Salmonella serotypes, Shigella sonnei and Shigella flexneri. Centers for Disease Control and Prevention, Atlanta, GA, USA.

Dewaele I, Rasschaert G, Bertrand S, Wildemauwe C, Wattiau P, Imberechts $\mathrm{H}$, Herman L, Ducatelle R, De Reu K, Heyndrickx M. Molecular characterization of Salmonella Enteritidis: comparison of an optimized multi-locus variable-number of tandem repeat analysis (MLVA) and pulsed-field gel electrophoresis. Foodborne Pathog Dis 2012;9:885-95.

EFSA, 2013. The European Union summary report on trends and sources of zoonoses, zoonotic agents and food-borne outbreaks in 2011. EFSA J 11:3129.

EFSA, 2015. EU summary report on antimicrobial resistance in zoonotic and indicator bacteria from humans, animals and food in 2013. EFSA J 13:3991.

Galanis E, Lo Fo, Wong DM, Patrick ME, Binsztein N, Cieslik A, Chalermchikit T, Aidara-Kane A, Ellis A, Angulo FJ, Wegener HC, World Health Organization Global Salmonella-Surveillance, 2006. Web-based surveillance and global Salmonella distribuition, 2000-2002. Emerg Infect Dis 12:381-8.

Grimont PAD, Weill FX, 2007. Antigenic formulae of the Salmonella serovars, 9th revision. WHO Collaborating Center for Reference and Research on Salmonella, Institut Pasteur, Paris, France.

Herikstad H, Motarjemi Y, Tauxe RV, 2002. Salmonella surveillance: a global survey of public health serotyping. Epidemiol Infect 129:1-8.

International Food Information Council Foundation, 2014. A consumer's guide to food safety risks. International Food Information Council Foundation, Washington, DC, USA.

ISO, 2004. Microbiology of food and animal feeding stuffs: horizontal method for the detection of Salmonella spp. ISO Norm 6579:2002/COR 1:2004. International Organization for Standardization, Geneva, Switzerland.

Kauffmann F, 1966. Classificazione e nomenclatura dei sub-genus I e IV delle Salmonelle. Zbl Bakt 202:484.

Le Minor L, Popoff MY, 1987. Designation of Salmonella enterica sp. as the type and only species of the genus Salmonella. Int J Syst Bacteriol 37:465.

Middleton D, Savage R, Tighe MK, Vrbova L, Walton R, Whitfield Y, Varga C, Lee B, Rosella L, Dhar B, Johnson C, Ahmed R, Allen VG, Crowcroft NS, 2014. Risk factors for sporadic domestically acquired Salmonella serovar Enteritidis infections: a case-control study in Ontario, Canada, 2011. Epidemiol Infect 142:1411-21.

Much P, Pichler J, Kasper S, Lassnig H, Kornschober C, Buchener A, Konig C, Allerberger F, 2009. A foodborne outbreak of Salmonella Enteritidis phage type 6 in Austria, 2008. Wien Klin Wochenschr 121:132-6.

Scallan E, Hoekstra RM, Angulo FJ, Tauxe RV, Widdowson MA, Roy SL, Jones JL, Griffin PM , 2011. Foodborne illness acquired in the United States-major pathogens. Emerg Infect Dis 17:7-15.

Shariat N, Sandt CH, DiMarzio MJ, Barrangou R, Dudley EG, 2013. CRISPR-MVLST subtyping of Salmonella enterica subsp. Enterica serovars Typhimurium and 
Heidelberg and application in identifying outbreak isolates. BMC Microbiol 13:254.

US Department of Agriculture, Economic Research Service, 2011. Foodborne illness cost calculator: Salmonella. US Department of Agriculture, Economic Research Service, Washington, DC, USA.
Available

from:

http://www.ers.usda.gov/data/foodborneilIness/salm_intro.asp

Zou W, Chen HC, Hise KB, Tang H, Foley SL, Meehan J, Lin WJ, Nayak R, Xu J, Fang H, Chen JJ, 2013. Meta-analysis of pulsedfield gel electrophoresis fingerprints based on a constructed salmonella database. PLoS One 8:e59224.

Zou W, Lin WJ, Foley SL, Chen CH, Nayak R, Chen JJ, 2010. Evaluation of pulsed-field gel electrophoresis profiles for identification of Salmonella serotypes. J Clin Microbiol 48:3122-6. 